\title{
Assessment of two hand hygiene regimens for intensive care unit personnel
}

\author{
Elaine L. Larson, RN, PhD, FAAN, CIC; Allison E. Aiello, MSPH; Jed Bastyr, BS; Chris Lyle, MS; \\ Julie Stahl, BS; Alicia Cronquist, RN, MPH; Lena Lai, BA; Phyllis Della-Latta, PhD, ABMM, FAMM
}

Objective: To compare skin condition and skin microbiology among intensive care unit personnel using one of two randomly assigned hand hygiene regimens: a $2 \%$ chlorhexidine gluconate (CHG)-containing traditional antiseptic wash and a waterless handrub containing $61 \%$ ethanol with emollients (ALC).

Design: Prospective, randomized clinical trial.

Setting: Two critical care units (medical and surgical) in a large, metropolitan academic health center in Manhattan.

Subjects: Fifty staff members (physicians, nurses, housekeepers, respiratory therapists) working full time in the intensive care unit.

Interventions: One of two hand hygiene regimens randomly assigned for four consecutive weeks.

Measurements and Main Results: The two outcomes were skin condition (measured by two tools: Hand Skin Assessment form and Visual Skin Scaling form) and skin microbiology. Samples were obtained at baseline, on day 1, and at the end of wks 2 and 4. Participants in the ALC group had significant improvements in the Hand Skin Assessment scores at wk $4(p=0.04)$ and in Visual Skin Scaling scores at wks $3(p=0.01)$ and $4(p=0.0005)$. There were no significant differences in numbers of colony-forming units between participants in the CHG or ALC group at any time period. The ALC regimen required significantly less time than the CHG regimen (mean: 12.7 secs and 21.1 secs, respectively; $p=$ 0.000 ) and resulted in a $50 \%$ reduction in material costs.

Conclusions: Changes in hand hygiene practices in acute care settings from the traditional antiseptic wash to use of plain, mild soap and an alcohol-based product should be considered. Further research is needed to examine the association between use of antiseptic products for hand hygiene of staff and reductions in nosocomial infection rates among patients. (Crit Care Med 2001; 29:944-951)

KeY WoRDS: antisepsis; handwashing; infection control; nosocomial infections; critical care; skin; hygiene; alcohol; chlorhexidine gluconate
T

he hands of healthcare personnel frequently serve as vectors for the transmission of organisms between patients. Although hand hygiene is one of the most important interventions in reducing transmission of potentially infectious agents, healthcare professionals wash less often and for shorter durations than recommended, and efforts to change their behavior have not been effective. Therefore, it is important to develop new approaches to improve hand hygiene. There is no national standard for hand hygiene products, but antiseptic detergents are most commonly used in adult and pediatric critical care settings (1).

From the Columbia University School of Nursing, New York, NY (E.L.L.); the Columbia University School of Public Health, New York, NY (A.E.A., A.C.); 3M Health Care, St. Paul, MN (J.B., C.L., J.S.); Columbia Presbyterian Medical Center, Clinical Microbiology Laboratory, New York, NY (L.L.); and the Department of Clinical Pathology, Columbia University Medical Center, New York, NY (P.D.-L.). MN.

Supported, in part, by 3M Health Care, St. Paul,

Copyright $\odot 2001$ by Lippincott Williams \& Wilkins
The need for frequent handwashing and the glove changing required among staff in high-risk, high-volume patient care areas such as intensive care units (ICUs) causes skin damage and dermatological problems among staff who practice appropriate hand hygiene vigorously. This skin damage leads to changes in normal bacterial hand flora, shedding of more organisms into the environment, and potentially increased risk of nosocomial transmission of pathogens from the hands of personnel to patients (1-3). The challenge is therefore to maximize the antimicrobial effectiveness of hand hygiene practices while minimizing changes to skin health or microflora in an effort to reduce nosocomial infection rates. The purpose of this study was to compare the effects of two hand-care regimens-a 2\% chlorhexidine gluconate (CHG)-containing traditional antiseptic wash (Foam Care, Ballard, Draper, UT) and a waterless handrub containing $61 \%$ ethanol with emollients (ALC) (Avagard, 3M Health Care, St. Paul, MN) - on skin microbiology and skin condition among ICU personnel.

\section{MATERIALS AND METHODS}

This was a 4-wk prospective, randomized clinical trial.

\section{Subjects and Setting}

Subjects were eligible for the study if they worked full time (>30 hrs/wk) in the medical or surgical ICU of a large medical center in northern Manhattan, were aged 18-65, were free from known allergy to study products, were not currently receiving topical or systemic steroids or antibiotics, and had no diagnosed current dermatologic conditions such as psoriasis. Subjects who reported a latex allergy were eligible for the study if they refrained from using latex products during the study.

The sample size was determined by the numbers required to detect a difference in the Visual Skin Scaling form (see description below). Previous studies have provided a SD estimate of $1.2(4,5)$. To have $80 \%$ power to detect a difference of 1 on the scale at a 0.05 level of significance, 19 subjects were required per group (6). To account for potential dropouts and poststudy exclusions, 25 subjects were recruited into each treatment group. 


\section{Instruments}

Assessment of Skin Condition. Although there are physiologic techniques to assess skin condition, many are not practical or reliable in clinical settings. These techniques include measurement of transepidermal water loss, electrical impedence, image analysis, and corneocyte shedding (7-9). Some tests require subjects to be in a resting metabolic state in a controlled environment for prolonged periods of time, so subjects would have to leave the ICU and go to a testing site. To address these limitations, several noninvasive tools with high reliability and validity to measure skin condition and irritant contact dermatitis have been developed and tested over the past $15 \mathrm{yrs}$, and are used in this study:

The Visual Skin Scaling (VSS) form, originally developed by Highley (10), is used to determine the amount of skin moisture and overall skin damage. The VSS is a six-point scale using stereomicroscopic examination of the hands at three times magnification, and correlates well with other physiologic measures of skin condition (10). The scores range from 6 (normal, no observable scale or irritation) to 0 (extensive cracking of skin surface, widespread reddening or occasional bleeding). In previous studies, including validation with dermatologist ratings (2-4), as well as in our pilot work for this study, an interrater agreement of $>95 \%$ within a score \pm 1 was consistently obtained over a large spectrum of damaged and undamaged hands of various skin types.

The Hand Skin Assessment (HSA) form is a self-rating scale developed in the 1980s for subjects to assess the condition of their hands. Subjects give themselves a score from 1 to 7 in four dimensions on their dominant hand: appearance, intactness, moisture content, and sensation. The possible range of scores is $4-28$, with 28 indicating totally healthy skin. In previous studies, scores correlated significantly with other physiologic measures of skin damage $(11,12)$. Previous studies have confirmed that self-reported symptoms combined with objective clinical measures of skin irritation such as the VSS, described above, provide valid and reliable measures of skin condition of hands that are reproducible and have a high level of sensitivity and specificity (13).

Diary Recordings. To assess the potential confounding effects of gloving and use of lotion, frequency of handwashing, and numbers of patient contacts, a diary card was kept by each subject to record the number of hand hygiene regimens performed, number of patients contacted, the number of glovings, and approximate total time spent in gloves (in hours) per working shift. Participants were asked to complete the diary card for ten working days ( $120 \mathrm{hrs}$ ) throughout the study. Reliability and validity of diary recordings were assessed by daily visits to participants on each shift at unexpected intervals, including random inspection of diary cards in progress. If staff had incomplete cards at any time during their shift, they were asked to refrain from completing the cards from memory and to start a new diary recording at the beginning of their next working shift. At the end of the study period, subjects assigned to the ALC protocol completed a questionnaire comparing the ALC and CHG protocols (all participants had been using the CHG protocol for at least 4 months before this study) for ease and speed of use, effect on skin and gloving, and preference.

Microbiological Techniques. Hands were sampled using a modified glove-juice technique. The subject inserted the dominant hand into a sterile polyethylene bag containing $50 \mathrm{~mL}$ of sampling solution $(0.075 \mathrm{M}$ phosphate buffer, $\mathrm{pH} 7.9$, containing $3 \%$ polysorbate $80 ; 0.1 \%$ Triton X-100; and $0.3 \%$ lecithin). This solution neutralizes any residual antiseptic on the skin and facilitates removal of microorganisms by dispersing the colonies into single cells, which can then be counted as colony-forming units (CFU). Before the study, the neutralizing system was validated to demonstrate that there was no effect of the sampling solution on growth of microorganisms and that antimicrobial activity was effectively neutralized (14). The entire hand was massaged by the data collector through the wall of the bag for $1 \mathrm{~min}$ and samples were taken to the microbiology laboratory within $1 \mathrm{hr}$ for processing. Before the first (baseline) sample, participants washed for 10 secs with a mild, nonantimicrobial soap to remove soil and transient contaminants. All subsequent cultures were obtained immediately after use of the assigned test product.

For the lowest limit of detection, $1.0 \mathrm{~mL}$ of the undiluted glove juice sample was spread plated across three plates $(0.3 \mathrm{~mL}, 0.3 \mathrm{~mL}$, and $0.4 \mathrm{~mL}$ ) of the following media (all from Becton Dickinson Microbiology Systems, Sparks, MD): 1) sheep blood agar (SBA) and colistinnalidixic acid (CNA), in duplicate, and 2) MacConkey, Sabouraud's (with chloramphenicol and gentamicin) and bile esculin, singly plated. The counts were added from all three plate dilutions of SBA and CNA to obtain the lowest limit of detection $\left(10^{\circ}\right)$ and an average was obtained from the duplicate series. This number was multiplied by $50 \mathrm{~mL}$ to produce the total CFU/hand. An inoculum of $0.1 \mathrm{~mL}$ of sampling solution (undiluted, 1:10, 1:100, and 1:1000) of glove juice sample was also spread plated in duplicate onto SBA and CNA.

All plates were incubated at $35^{\circ} \mathrm{C}$ and observed daily for growth over $48 \mathrm{hrs}$ for bacteria and up to 7 days for yeast. Speciation of Gramnegative bacilli was performed using Api 20 NE and Api 20E (bioMerieux, Hazelwood, MO), staphylococci by coagulase and Staphaurex (Murex Biotech Limited, Norcross, GA), enterococci and micrococci by MicroScan (Dade Behring, Deerfield, IL), and yeast by germ tube formation.

Procedures. The study protocol was reviewed and approved by the institutional re- view board representing the study institution, and subjects signed a written consent form before enrollment. Persons who volunteered and met all inclusion and none of the exclusion criteria were assigned randomly generated subject numbers to determine their treatment group assignment. Upon enrollment in the study, the subjects completed a demographic form and provided information regarding their usual hand hygiene practices and the usual condition of their hands. Subjects in the ALC group received training in the application procedure. They were provided with individual bottles of the ALC product and a mild, nonantimicrobial liquid soap, with instructions to use the soap only if hands became soiled or needed physical cleaning. They were instructed to dispense sufficient product into their palms to moisten the entire surface of the hands, about 2-3 mL. Subjects using $\mathrm{CHG}$, which was the current product in use in both study units, were reminded to use the product according to the directions provided by the manufacturer on each container. The skin condition measures (VSS and HSA) and hand cultures were obtained four times: at baseline, during the first day of wk 1 , and as late as possible on the subject's last workday of wks 2 and 4.

Because the use of hand lotion can affect the primary outcome of skin condition, and many common lotions inactivate CHG (15, $16)$, all subjects were provided with a CHGcompatible lotion (Prevacare, Johnson \& Johnson, Arlington, TX). Hand lotion usage was measured in two ways: subjects were asked to record each application of lotion on their diary cards, and the bottles were weighed (in grams) at the end of the study to determine the volume used. We made every attempt to ease the participant burden with convenient signs, recording sheets, and in-person reminders.

Over the course of the study, 69 observations of participants' hand hygiene practices were made at various times throughout the working period. The time required to perform the hand hygiene regimen and deviations from the prescribed protocol (e.g., touching the sink area immediately after washing, failing to cover all hand surfaces or to dry hands) were recorded for both treatment groups. The observations were made in the last 3 wks of the treatment period (wks 2-4) after study subjects had been using the product long enough to have established a comfortable routine of application. Over the study period, one or more members of the research team made daily visits on all shifts to monitor compliance to the study protocol, answer questions, and collect data.

\section{Data Analysis}

All bacterial counts were transformed to $\log$ base $_{10}$ before analysis to normalize the data. The Kendall tau-b, chi-square, or Fisher's exact test was used to compare differences 
in proportions between the ALC and CHG groups in categorical variables. A "change score" was calculated to examine the measure of skin condition by subtracting the score at each measurement interval with the baseline score (e.g., VSS2-VSS1). The nonparametric Mann-Whitney test was then used to compare these change scores for VSS and HSA between the two groups. The Student's $t$-test was used to compare the two groups with regard to continuous variables. Analysis of covariance was used to compare log CFU counts between the two groups at each time interval, controlling for baseline counts. The paired Student's $t$-test was used to compare microbial counts at each time interval (day 1, wk 2, wk 4) with baseline counts within the ALC and CHG groups separately. A hand hygiene ratio was calculated (number of hand hygiene episodes/ number of patients contacted) and compared using the nonparametric Kruskal-Wallis test across disciplines (physicians, nurses, other staff). One-sided $p$ values were calculated for the skin condition variables; two-sided tests were used for all other variables. A cost-in-use calculation was also done using the acquisition cost of each product and the usage volume specified in each manufacturer's directions for use.

\section{RESULTS}

Fifty subjects entered the study; half each in the ALC and CHG groups. Fortysix subjects completed the entire study. Two subjects discontinued participation because they did not choose to continue with data collection, one left the study unit before the final sample, and one nurse in the ALC group had a dermatologic problem that required dropping out of the study. Follow-up on that problem revealed that the participant had a history of multiple allergies, and later patch testing for potential allergy to ingredients in the study product was negative. There were no significant differences between the two treatment groups with regard to any demographic or clinical practice variables (Table 1).

\section{Reported Skin Care Practices}

Participants recorded an average of 116.9 hrs of time in their hand hygiene diaries. Mean reported handwashes/12-hr shift for the entire group was 11.7 and mean ALC applications was 17.7. Lotion was applied a mean of 5.5 times, and participants reported contact with an average of 3.96 patients/shift. An average of 15.7 glovings were reported, with an average number of hours in gloves esti-

Table 1. Characteristics of study population

\begin{tabular}{|c|c|c|c|}
\hline Characteristic & ALC Group & CHG Group & $p$ Value \\
\hline \multicolumn{4}{|l|}{ Unit } \\
\hline MICU & 12 & 16 & \multirow[t]{2}{*}{$0.12^{a}$} \\
\hline SICU & 14 & 8 & \\
\hline \multicolumn{4}{|l|}{ Profession } \\
\hline Physician & 2 & 5 & \multirow{3}{*}{$0.58^{b}$} \\
\hline Nurse & 21 & 15 & \\
\hline Other staff & 3 & 4 & \\
\hline \multicolumn{4}{|l|}{ Gender } \\
\hline Female & 20 & 19 & \multirow[t]{2}{*}{$0.56^{a}$} \\
\hline Male & 6 & 5 & \\
\hline \multicolumn{4}{|l|}{ Dominant hand } \\
\hline Right & 25 & 20 & \multirow[t]{2}{*}{$0.18^{a}$} \\
\hline Left & 1 & 4 & \\
\hline \multicolumn{4}{|l|}{ Ethnicity } \\
\hline African-American & 5 & 6 & \multirow{3}{*}{$0.26^{b}$} \\
\hline Hispanic & 3 & 0 & \\
\hline White & 9 & 4 & \\
\hline Asian & 9 & 14 & \\
\hline \multicolumn{4}{|l|}{ Wear artificial nails? } \\
\hline Never & 24 & 21 & \multirow[t]{2}{*}{$0.58^{b}$} \\
\hline Sometimes & 2 & 3 & \\
\hline \multicolumn{4}{|l|}{ Wear nail polish? } \\
\hline Never & 14 & 12 & \multirow[t]{2}{*}{$0.77^{b}$} \\
\hline Sometimes/routinely & 12 & 12 & \\
\hline \multicolumn{4}{|l|}{ Use lotion? } \\
\hline$<3$ times/day & 14 & 14 & \multirow[b]{2}{*}{$0.82^{b}$} \\
\hline$>3$ times/day & 12 & 10 & \\
\hline \multicolumn{4}{|l|}{ Type of gloves } \\
\hline Any type provided & 20 & 16 & \multirow{4}{*}{$0.97^{b}$} \\
\hline Latex powder-free only & 1 & 2 & \\
\hline Nonlatex powder-free only & 5 & 5 & \\
\hline Any powder-free & 0 & 1 & \\
\hline Estimated time in gloves/shift & & & \\
\hline$<1 \mathrm{hr}$ & 3 & 3 & \\
\hline $1-2 \mathrm{hrs}$ & 3 & 4 & $0.34^{b}$ \\
\hline $3-4$ hrs & 4 & 5 & \\
\hline$>4 \mathrm{hrs}$ & 16 & 12 & \\
\hline Problems with hands? & & & \\
\hline Never & 6 & 6 & \\
\hline Rarely/occasionally & 12 & 12 & $0.57^{b}$ \\
\hline Frequently/persistently & 8 & 6 & \\
\hline Severity of problems & & & \\
\hline Not applicable & 8 & 7 & \\
\hline Not too serious & 9 & 12 & $0.54^{b}$ \\
\hline Worse than most & 8 & 5 & \\
\hline Very serious & 1 & 0 & \\
\hline Age & & & \\
\hline Mean & 40.5 & 40.6 & \\
\hline Range & $30-60$ & $29-57$ & $0.96^{c}$ \\
\hline SD & 7.28 & 6.95 & \\
\hline Years in current position & & & \\
\hline Mean & 10.7 & 8.5 & \\
\hline Range & $1-28$ & $2-25$ & $0.28^{c}$ \\
\hline SD & 8.20 & 5.93 & \\
\hline Years in profession & & & \\
\hline Mean & 16.1 & 14.0 & \\
\hline Range & $1.5-28$ & $2-31$ & $0.28^{c}$ \\
\hline SD & 6.70 & 6.48 & \\
\hline
\end{tabular}

$A L C$, waterless handrub containing $61 \%$ ethanol with emollients; $C H G, 2 \%$ chlorhexidine gluconate-containing traditional antiseptic wash; MICU, medical intensive care unit; SICU, surgical intensive care unit.

${ }^{a}$ Fisher's exact test.

${ }^{b}$ Kendall's tau-b.

${ }^{c}$ Independent samples $t$-test.

mated to be 2.6 hrs each shift. There was a significant correlation between the selfreported number of applications of lotion and the amount of lotion used as verified by weighing the lotion bottles at the end of the study $(p<0.001)$. As expected, the 
CHG group reported significantly more handwashes/shift, inasmuch as the ALC group was instructed not to wash their hands unless they were physically soiled. There were no significant differences in other practices between the two hand hygiene groups (Table 2).

There were no significant differences by profession (physician, nurse, or other staff) in reported total mean hours in gloves/shift $(p=0.09)$, but nurses and other staff donned gloves about three times more often than did physicians ( $p$ $=0.01$ ). Whereas nurses and other staff reported a greater number of hand hygiene episodes (handwashes plus alcohol applications) per shift than physicians ( $p$ $=0.21$ ), nurses and other staff also touched patients more often than did physicians $(p=0.02)$. Hence, the hand hygiene ratio (hand hygiene episodes/ number of different patients touched) was significantly higher for physicians than for nurses $(p=0.001)$, i.e., they washed more often per number of different patients contacted. Although reported mean number of lotion applications did not differ significantly by discipline $(p=0.15)$, other staff (respiratory therapists and housekeepers) used a significantly larger volume of lotion than did physicians or nurses $(p=0.04)$ (Fig. $1)$.

\section{Skin Condition}

There were no significant differences in either of the skin assessment measures at baseline or during the first day between the two study groups. All change scores (difference from baseline) for the ALC group showed improvement, and all change scores for the CHG group except at wk 2 showed a worsening of skin condition. At wks 3 and 4, those in the ALC group had significantly better VSS scores $(p=0.01$ and $p=0.0005$, Table 3$)$. By wk 4 , subjects' self-assessment of skin condition using the HSA was also significantly better for those in the ALC group ( $p=$ $0.04)$. Although there was no significant difference between the two groups at baseline in the subscale of "moisture" $(p$ $=0.36$ ), by wk 4 those in the ALC group scored significantly better (mean scores 6.26 and 4.74, respectively; $p=0.02$ ). Subjects in the CHG group applied lotion $50 \%$ more often than subjects in the ALC group (means of 4.3 vs. 6.7 applications/ day, respectively; $p=0.19$ ).
Table 2. Hand hygiene practices reported by study participants

\begin{tabular}{|c|c|c|c|}
\hline Practice & ALC Group & CHG Group & $\begin{array}{c}p \text { Value } \\
\text { (Student's } t \text {-test) }\end{array}$ \\
\hline \multicolumn{4}{|l|}{ Diary hours recorded } \\
\hline Mean & 115.5 & 118.5 & \multirow{3}{*}{0.69} \\
\hline Range & $48-144$ & $0-180$ & \\
\hline $\mathrm{SD}$ & 22.88 & 27.55 & \\
\hline \multicolumn{4}{|l|}{ Handwashes/shift } \\
\hline Mean & 6.1 & 16.7 & \multirow{3}{*}{0.001} \\
\hline Range & $0-39$ & $2-37$ & \\
\hline $\mathrm{SD}$ & 10.2 & 9.4 & \\
\hline \multicolumn{4}{|l|}{ ALC applications/shift } \\
\hline Mean & 17.7 & NA & \multirow{3}{*}{ NA } \\
\hline Range & $4-43$ & & \\
\hline $\mathrm{SD}$ & 9.8 & & \\
\hline \multicolumn{4}{|c|}{ Lotion applications/shift } \\
\hline Mean & 4.3 & 6.7 & \multirow{3}{*}{0.19} \\
\hline Range & $0-23$ & $0-23$ & \\
\hline $\mathrm{SD}$ & 5.64 & 6.15 & \\
\hline \multicolumn{4}{|c|}{ Amount of lotion used, grams } \\
\hline Mean & 39.6 & 38 & \multirow{3}{*}{0.91} \\
\hline Range & $0-150$ & $0-150$ & \\
\hline $\mathrm{SD}$ & 55.10 & 40.91 & \\
\hline \multicolumn{4}{|l|}{ Glovings/shift } \\
\hline Mean & 15.5 & 16.0 & \multirow{3}{*}{0.81} \\
\hline Range & $1-46$ & $3-37$ & \\
\hline $\mathrm{SD}$ & 9.82 & 8.76 & \\
\hline \multicolumn{4}{|l|}{ Hours/shift in gloves } \\
\hline Mean & 2.1 & 2.9 & \multirow{3}{*}{0.24} \\
\hline Range & $0.3-5$ & $0.5-10.5$ & \\
\hline $\mathrm{SD}$ & 1.19 & 2.65 & \\
\hline \multicolumn{4}{|c|}{ Patients contacted/shift } \\
\hline Mean & 3.5 & 4.4 & \multirow{3}{*}{0.24} \\
\hline Range & $0-7$ & $1-11$ & \\
\hline SD & 1.54 & 2.73 & \\
\hline
\end{tabular}

$A L C$, waterless handrub containing $61 \%$ ethanol with emollients; $C H G, 2 \%$ chlorhexidine gluconate-containing traditional antiseptic wash; NA, not applicable.

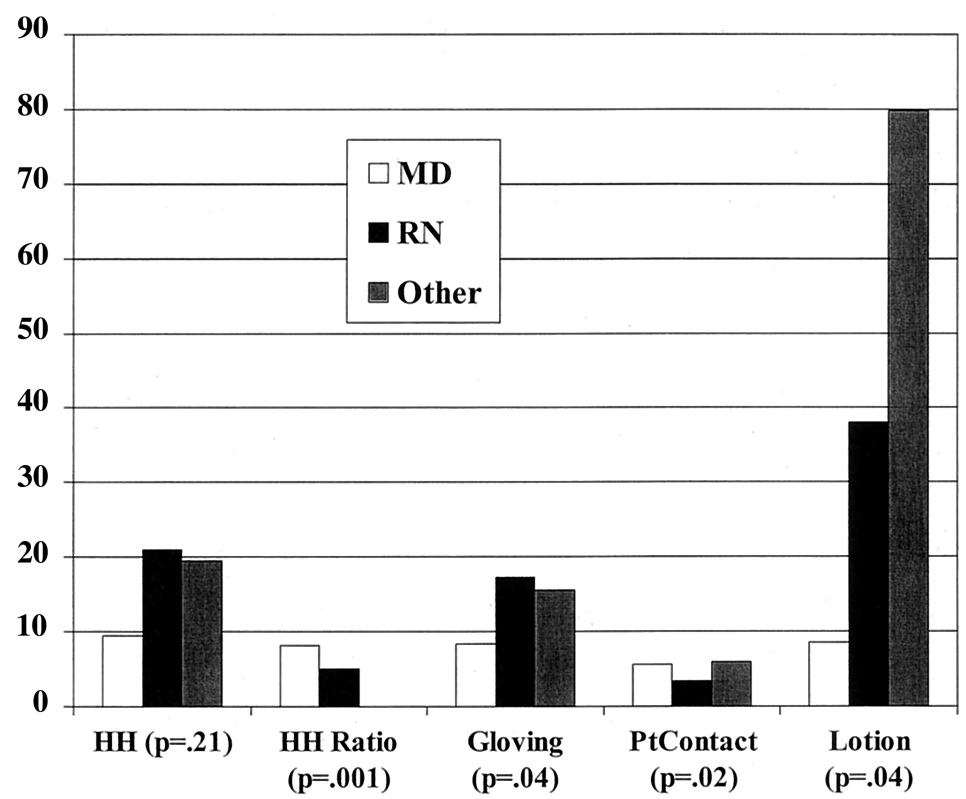

Figure 1. Differences in practices by discipline ( 7 physicians $[M D], 35$ nurses $[R N]$, and 7 other staff members). $H H$, mean number of hand hygiene episodes/12-hr working shift (traditional handwashes + applications of ALC); HH Ratio, mean number of hand hygiene episodes/number of patients contacts/12-hr shift; Gloving, mean number of times gloves were donned/12-hr shift; PtContact, mean number of individual patients touched/12-hr shift; Lotion, mean number of applications of lotion/ 12-hr shift. 


\section{Skin Microbiology}

In the 193 hand cultures obtained, all subjects had coagulase-negative staphylococci isolated from hands, and there were 13 isolates of methicillin-sensitive Staphylococcus aureus. In addition, there were 40 isolates of Gram-negative bacteria (Acinetobacter, Pseudomonas, Serratia, Klebsiella, Enterobacter spp.), 21 in the ALC group, and 19 in the CHG group ( $p$ $=0.64$ ); and 30 isolates of Candida spp., 9 in the ALC group and 21 in CHG group $(p=0.02)$. No methicillin-resistant $S$. aureus or vancomycin-resistant enterococci were isolated.

Participants in the ALC group had significantly higher log CFU counts at baseline than the CHG group (mean log counts 5.03 and 4.41, respectively; $p=$ 0.01 ), but there were no significant differences in CFU counts between the two groups at any other time interval after controlling for baseline counts with analysis of covariance. For the CHG group, there were no significant differences between baseline mean log counts and mean $\log$ counts from day 1 , wk 2 , or wk 4 . For the ALC group, counts were significantly lower than baseline at day 1 (5.03 vs. 4.59 , respectively; $p=0.03$ ), but not at other time points. The microbial counts on hands of participants using CHG increased slightly in wks 2 and 4, whereas the counts decreased slightly at each time interval among those using ALC (Table 4).

\section{Participants' Practices and Assessment of Products}

Observations of participants' hand hygiene practices included $36(62.2 \%)$ in the medical ICU and $33(47.8 \%)$ in surgical ICU, 41 (59.4\%) on the day shift and $28(40.6 \%)$ on nights, and $38(55.1 \%)$ with the ALC protocol and 31 (44.9\%) on $\mathrm{CHG}$ protocol. The ALC product and regimen resulted in a $41 \%$ decrease in application time, from a mean of 21.5 secs for CHG to a mean of 12.7 secs for ALC ( $p=$ $0.0001)$. The ALC also resulted in less deviation from protocol, with $7(22.6 \%)$ of CHG observations showing deficiencies in practice vs. 3 (7.9\%) for ALC (e.g., recontamination of hands, failure to cover entire surface, failure to dry hands; Fishers' exact test, $p=0.08$ ). Based on manufacturer's directions for use and cost of each product, the ALC product reduced the cost from \$0.05/application with $\mathrm{CHG}$ to $\$ 0.025 /$ application, an over-

Table 3. Skin condition of participants

\begin{tabular}{|c|c|c|c|}
\hline Measurement & ALC Group & CHG Group & $\begin{array}{c}p \text { Value } \\
\text { (Mann-Whitney) }\end{array}$ \\
\hline \multicolumn{4}{|l|}{$\mathrm{HSA}^{a}{ }^{a}$ Baseline } \\
\hline Mean & 22.7 & 23.3 & \multirow{3}{*}{0.44} \\
\hline Range & $10-28$ & $16-28$ & \\
\hline $\mathrm{SD}$ & 5.45 & 3.59 & \\
\hline \multicolumn{4}{|l|}{ HSA, mid-day 1} \\
\hline Mean & 25.1 & 23.3 & \multirow{3}{*}{0.11} \\
\hline Range & $12-28$ & $12-28$ & \\
\hline $\mathrm{SD}$ & 3.81 & 4.73 & \\
\hline \multicolumn{4}{|l|}{ HSA, wk 2} \\
\hline Mean & 24.9 & 23.5 & \multirow{3}{*}{0.09} \\
\hline Range & $15-28$ & $15-28$ & \\
\hline $\mathrm{SD}$ & 3.4 & 3.83 & \\
\hline \multicolumn{4}{|l|}{ HSA, wk 4} \\
\hline Mean & 25.4 & 22.8 & \multirow{3}{*}{0.04} \\
\hline Range & $17-28$ & $12-28$ & \\
\hline $\mathrm{SD}$ & 2.76 & 5.44 & \\
\hline \multicolumn{4}{|l|}{$\mathrm{VSS}^{b}$ baseline } \\
\hline Mean & 5.08 & 5.25 & \multirow{3}{*}{0.35} \\
\hline Range & $2-6$ & $3-6$ & \\
\hline SD & 1.08 & 0.85 & \\
\hline \multicolumn{4}{|l|}{ VSS, mid-day 1} \\
\hline Mean & 5.37 & 5.17 & \multirow{3}{*}{0.06} \\
\hline Range & $2-6$ & $2-6$ & \\
\hline $\mathrm{SD}$ & 0.97 & 1.01 & \\
\hline \multicolumn{4}{|l|}{ VSS, wk 2} \\
\hline Mean & 5.50 & 4.95 & \multirow{3}{*}{0.01} \\
\hline Range & $4-6$ & $3-6$ & \\
\hline SD & 0.72 & 0.95 & \\
\hline \multicolumn{4}{|l|}{ VSS, wk 4} \\
\hline Mean & 5.65 & 4.95 & \multirow{3}{*}{0.0005} \\
\hline Range & $4-6$ & $3-6$ & \\
\hline $\mathrm{SD}$ & 0.65 & 0.98 & \\
\hline
\end{tabular}

$A L C$, waterless handrub containing $61 \%$ ethanol with emollients; $C H G, 2 \%$ chlorhexidine gluconate-containing traditional antiseptic wash; HSA, Hand Skin Assessment; VSS, Visual Skin Scaling.

${ }^{a}$ HSA form, subject assessment (range: $4-28$ ).

${ }^{b}$ VS Skin condition, observer assessment (range: 1-6).

Table 4. Microbial counts on hands of participants $\left(\log _{10} \mathrm{CFU}\right)$

\begin{tabular}{lccc}
\hline A. Differences between ALC and CHG groups & & \\
\hline Time Interval & ALC Group (+/- SE) & CHG Group (+/- SE) & $p$ Value \\
\hline Baseline & $5.03(0.15)$ & $4.41(0.19)$ & 0.02 \\
Mid-day 1 & $4.64(0.17)$ & $4.47(0.17)$ & $0.56^{a}$ \\
Wk 2 & $4.59(0.19)$ & $4.50(0.16)$ & $0.20^{a}$ \\
Wk 4 & $4.72(0.19)$ & $4.64(0.17)$ & $0.40^{a}$ \\
\hline
\end{tabular}

B. Differences between baseline and subsequent cultures for ALC and CHG groups

$\log _{10}$ CFU Difference from Baseline

\begin{tabular}{lcccccc}
\cline { 2 - 6 } Group & Mid-Day 1 & $p$ Value $^{b}$ & Wk 2 & $p$ Value $^{b}$ & Wk 4 & $p$ Value $^{b}$ \\
\hline ALC & $-0.43 \log$ & 0.03 & $-0.46 \log$ & 0.04 & $-0.31 \log$ & 0.18 \\
CHG & $+0.07 \log$ & 0.69 & $+0.09 \log$ & 0.59 & $+0.24 \log$ & 0.12 \\
\hline
\end{tabular}

$A L C$, waterless handrub containing $61 \%$ ethanol with emollients; $C H G, 2 \%$ chlorhexidine gluconate-containing traditional antiseptic wash; $C F U$, colony-forming units.

${ }^{a}$ Analysis of covariance.

${ }^{b}$ Paired $t$-test.

all cost savings of $50 \%$ for materials. Further cost savings would be recognized for ALC if time savings were also factored in. Participants rated the ALC regimen easier
(75\%), faster $(90 \%)$, and milder $(80 \%)$, and expressed a preference for the regimen $(70 \%)$ when compared with the CHG regimen (Fig. 2). 


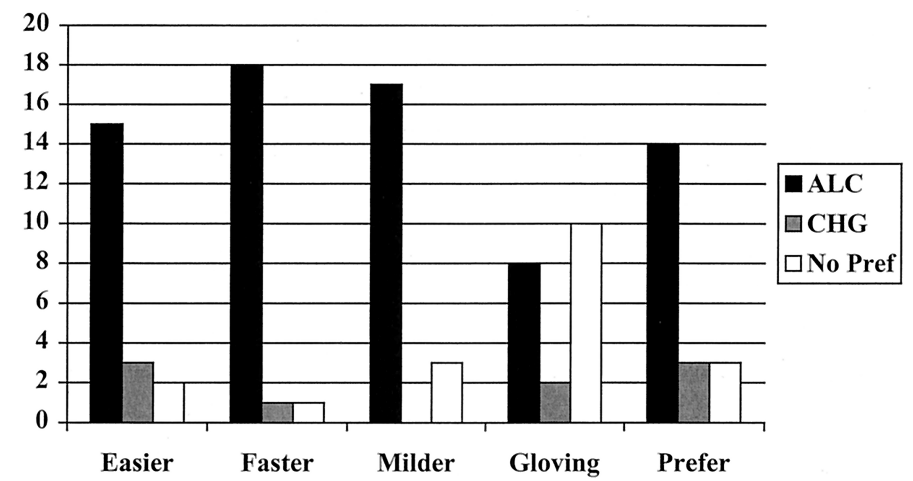

Figure 2. Number of participants who preferred alcohol $(A L C)$, chlorhexidine $(\mathrm{CHG})$, or neither (No Pref). Easier, Which product is easier to use? Faster, Which product is faster to use? Milder, Which product is milder on your hands? Gloving, Which product makes it easier to don gloves? Prefer, Overall, which product do you prefer?

\section{DISCUSSION}

Differences in hand hygiene practices among physicians and nurses have been frequently described $(3,17-21)$, and our findings in that regard are consistent with others. Although critical care nurses touch only a few patients during each working shift, they are in frequent and close contact with them, performing a variety of procedures that place them at high risk for acquisition and transmission of patient flora. Hence, it is often necessary that they wash their hands and they don gloves more often than physicians. The frequency of handwashing and gloving were two variables significantly predictive of skin damage in a previous study (2), so it is not surprising that nurses also used more lotion than physicians. Skin damage has also been associated with undesirable changes in the microbial flora of the hands, and should be avoided $(22,23)$ by keeping the skin moisturized (24). With regard to our measurement tools for skin condition, the observer-completed VSS and participant-completed HSA were well correlated.

\section{Detergent-Based Antiseptics and Alcohols}

Efficacy and Effectiveness. In this study, a hand hygiene regimen that replaced use of a detergent-based antiseptic with a waterless lotion containing alcohol and emollient resulted in similar microbiological effectiveness and improved skin condition of critical care staff. These results are consistent with results of two additional studies that we have recently conducted in a neonatal ICU (25) and a surgical setting (5). In the former small pilot study (16 neonatal ICU nurses), the alcohol hand regimen resulted in comparable log CFU reductions and was associated with a significant improvement in skin condition when compared with the traditional antiseptic handwash regimen. In the operating room trial, an alcohol/ CHG product containing emollients replaced the traditional surgical scrub during a 3-wk crossover period and was again associated with significantly lower log CFU counts and significantly improved skin condition among 22 surgical personnel. It is important to note that the alcohol products used in each of these studies were not the same. Although improvement in skin condition and superior log reductions were associated with specially formulated products, a common ingredient was alcohol, indicating that it is possible to formulate alcohol products that are highly efficacious and mild to the skin. Although detergent-based antiseptic products are commonly used by staff in U.S. critical care settings, alcohols have been used for decades, particularly in several European countries, for hand hygiene, and their antimicrobial effectiveness and rapid activity are extensively documented (26-38).

In addition to antimicrobial effectiveness, two other criteria are important when selecting hand hygiene products for high-risk patient care areas-effects on skin health and cost. One major deterrent to the adoption of alcohol-based products in U.S. hospitals has been concerns about the potential damaging effects of alcohol on the skin. In fact, when Doebelling et al. (39) in 1992 conducted a crossover study in several ICUs to compare traditional handwashing with alcohol hand rinse, there was a significant reduction in frequency of hand hygiene during the alcohol phase-staff simply did not use it. Probably as a result of this confounder, the investigators reported a significant reduction in nosocomial infection rates during the period of use of antiseptic detergent. This was unfortunate, because there are essentially no other clinical trials that have assessed the impact of alcohol-based products vs. traditional handwashing on the outcome of nosocomial infections. The Doebelling study was of great importance because it demonstrated the added value of antiseptic handwashing, but the comparison product was primarily a plain, nonantimicrobial soap rather than alcohol.

Concerns about the drying and damaging effects of alcohol products seem to be unfounded. Data from recent studies consistently demonstrate that they are actually less damaging to the skin than soaps and detergents, and their use is associated with increased staff compliance with hand hygiene standards (28, $33,40-43)$. With regard to cost, Voss and Widmer (44) demonstrated that use of alcohol-based products in critical care areas would reduce the time required for hand hygiene by about $80 \%$. In our study, we confirmed that the ALC regimen took significantly less time than the traditional wash.

Potential Untoward Effects. No hand hygiene product is free from potential risks or side effects. Reported untoward effects of CHG include keratitis and corneal damage $(45,46)$, local skin reactions (47, 48), and anaphylaxis (49-53). Although alcohol has long been considered one of the safest antiseptics available, allergic contact dermatitis and urticaria have been reported following alcohol use (54-56). One nurse in the ALC arm did have a dermatologic reaction that required her to drop out of this study, but it was unclear whether this was associated with the product or with preexisting risk factors. Both CHG and alcohol aerosols have caused occupational asthma in nurses (57).

One disadvantage of antiseptic products containing $\mathrm{CHG}$ is that they are neutralized by anionic surfactants present in most over-the-counter hand lotions (15, 16). This requires that special lotions be provided for staff working in units in which CHG-containing products are used. A potential concern with alcoholcontaining products is flammability, but the only recently reported case of a problem related to topical application was as- 
$\mathrm{U}$ nder in-use conadult critical care hygiene product was comparable with a $2 \%$ chlorhexidine gluconate-containing antiseptic detergent in terms of antimicrobial effectiveness, was associated with improved skin condition, and took significantly less

time for use. ditions in two units, an alcohol-based hand

\section{SUMMARY AND CONCLUSIONS} critical care units, an alcohol-based hand hygiene product was comparable with a CHG-containing antiseptic detergent in terms of antimicrobial effectiveness, was associated with improved skin condition, and took significantly less time for use. These findings are consistent with a number of other studies, and indicate that it is appropriate to consider changes in hand hygiene practices in acute care settings from the traditional antiseptic wash to use of plain, mild soap for cleaning and an alcohol-based product for degerming. Further research is needed to examine the association between use of antiseptic products for hand hygiene of staff (both detergent and alcohol-based) and reductions in nosocomial infection rates among patients.

\section{ACKNOWLEDGMENTS} oration and support of the administrative staff of the surgical and medical ICUs, particularly J. Abramson, RN; E.M. Lazaro, RN, MEd; W.D. Roberts, RN, MSN; R. Sladen, MD; and I.S. Douglas, MD.

\section{REFERENCES} assure and monitor compliance with study protocols, and there were indeed deviations from the protocol. For example, some staff members reported forgetting their lotion and sharing with others, and some found the diary recording to be a burden and sometimes recorded their practices after several hours, depending on their recall. Generally, when their diary cards were checked, staff were found to be very conscientious about real-time recording. The fact that there was a significant correlation between amount of lotion used as measured by self-report and by weighing of lotion bottles was an indication that the reliability of staff reporting was satisfactory. Finally, our cost estimates were based on product volumes recommended by the manufacturer rather than actual volumes used because we thought it more appropriate and generalizable to use this standardized amount, and because our random observations of staff confirmed that the volume of product being used was generally in accordance with directions.
Under in-use conditions in two adult

We gratefully acknowledge the collab-

tergents as measured by skin water loss. Dermatology 1982; 164:314-321

9. McGinley KJ, Marples RR, Plewig G: A method for visualizing and quantitating the desquamating portion of the human stratum corneum. J Invest Dermatol 1969; 53: $107-111$

10. Highley DR, Savoyka VO, O'Neill JJ, et al: A stereomicroscopic method for the determination of moisturizing efficacy in humans. J Soc Cosmet Chem 1976; 27:351-363

11. Larson E, Leyden J, McGinley K, et al: Physiologic and microbiologic changes in skin related to frequent handwashing. Infect Control 1986; 7:59-63

12. Larson E, McGinley K, Grove G, et al: Physiologic, microbiologic and seasonal effects of handwashing on the skin of health care personnel. Am J Infect Control 1986; 14:51-59

13. Simion FA, Rhein LD, Morrison BM, et al: Self-perceived sensory responses to soap and synthetic detergent bars correlate with clinical signs of irritation. J Am Acad Dermatol 1995; 32:205-211

14. Sutton S: Neutralizer evaluations as control experiments for antimicrobial efficacy tests. In: Handbook of Disinfectants and Antiseptics. Ascenzi JM (Ed). New York: Marcel Dekker, 1996

15. Benson L, LeBlanc D, Bush L, et al: The effects of surfactant systems and moisturizing products on the residual activity of a chlorhexidine gluconate handwashing using a pigskin substrate. Infect Control Hosp Epidemiol 1990; 11:67-70

16. Frantz SW, Haines KA, Azar CG, et al: Chlorhexidine gluconate activity against clinical isolates of vancomycin-resistant Enterococcus faecium (VREF) and the effects of moisturizing agents on CHG residue accumulation on the skin. $J$ Hosp Infect 1997; 37: 157-164

17. Donowitz LG: Handwashing technique in a pediatric intensive care unit. Am J Dis Child 1987; 141:683-685

18. DeCarvalho M, Lopes JMA, Pellitteri M: Frequency and duration of handwashing in a neonatal intensive care unit. Pediatr Infect Dis $J$ 1989; 8:179-180

19. Meengs MR, Giles BK, Chisholm CD, et al: Hand washing frequency in an emergency department. J Emerg Nurs 1994; 20:183-188

4. Larson E, Friedman C, Cohran J, et al: Prevalence and correlates of skin damage on the hands of nurses. Heart Lung 1997; 26: 404-412

5. Larson EL, Aiello AE, Heilman JM, et.al: Comparison of traditional surgical scrub and brushless application of a waterless alcohol/ CHG lotion. AORN J 2000; 72:1-12

6. Zar J. Biostatistical Analysis. Second Edition. Englewood Cliffs, NJ: Prentice-Hall, 1984, p 153

7. Grove GL: Techniques for substantiating skin care product claims. In: Safety and Efficacy of Topically Applied Drugs and Cosmetics. Kligman AM, Leyden JJ (Eds). New York: Grune \& Stratton, 1982

8. Hassing JH, Nater JP, Bleumink: Irritancy of low concentrations of soap and synthetic de-
20. Dorsey ST, Cydulka RK, Emerman CL: Is handwashing teachable? Failure to improve handwashing behavior in an urban emergency department. Acad Emerg Med 1996; 3:360-365

21. Pittet D, Mourouga P, Perneger TV: Compliance with handwashing in a teaching hospital. Ann Intern Med 1999; 130:126-130

22. Larson EL, Hughes CAN, Pyrek JD, et al: Changes in bacterial flora associated with skin damage on hands of health care personnel. Am J Infect Control 1998; 26:513-522

23. Larson E: Skin hygiene and infection prevention: More of the same or different approaches? Clin Infect Dis 1999; 29: 1287-1294 
24. McCormick RA, Buchman T, Maki DG: Double-blind, randomized trial of scheduled use of a novel barrier cream and an oil-containing lotion for protecting the hands of health care workers. Am J Infect Control 2000; 28: 302-310

25. Larson E, Silberger M, Jakob K, et al: Assessment of alternative hand hygiene regimens to improve skin health among neonatal intensive care unit nurses. Heart Lung 2000; 29:136-142

26. Lilly HA, Lowbury EJL, Wilkins MD: Limits to progressive reduction of resident skin bacteria by disinfection. J Clin Pathol 1979; 32 : 382-385

27. Rotter ML: Hygienic hand disinfection. Infect Control 1984; 5:18-22

28. Larson EL, Eke PI, Laughon BE: Efficacy of alcohol-based hand rinses under frequentuse conditions. Antimicrob Agents Chemother 1985; 30:542-544

29. Morrison AJ, Gratz J, Cabezudo I, et al: The efficacy of several new handwashing agents for removing non-transient bacterial flora from hands. Infect Control 1986; 7:268-272

30. Rotter ML, Koller W, Wewalka G, et al: Evaluation of procedures for hygienic handdisinfection: Controlled parallel experiments on the Vienna test model. J Hyg 1986; 96: $27-37$

31. Eckert DG, Ehrenkranz NJ, Alfonso BC: Indications for alcohol or bland soap in removal of aerobic Gram-negative skin bacteria: Assessment by novel method. Infect Control Hosp Epidemiol 1989; 10:306-311

32. Larson E, Bobo L: Effective hand degerming in the presence of blood. J Emerg Med 1992; 10:7-11

33. Pereira LJ, Lee GM, Wade KJ: An evaluation of five protocols for surgical handwashing in relation to skin condition and microbial counts. J Hosp Infect 1997; 36:49-65

34. Deshmukh N, Kramer JW, Kjellberg SI: A comparison of 5-minute povidone-iodine scrub and 1-minute povidone-iodine scrub followed by alcohol foam. Mil Med 1998; 163: 145-147
35. Kampf G, Hofer M, Wendt C: Efficacy of hand disinfectants against vancomycin-resistant enterococci in vitro. J Hosp Infect 1999; 42: 143-150

36. Paulson DS, Fendler EJ, Dolan MJ, et al: A close look at alcohol gel as an antimicrobial sanitizing agent. Am J Infect Control 1999; 27:332-338

37. Zaragoza M, Salles M, Gomez J, et al: Handwashing with soap or alcoholic solutions? A randomized clinical trial of its effectiveness. Am J Infect Control 1999; 27:258-261

38. Jones RA, Jampani H, Mulberry G, et al: Moisturizing alcohol hand gels for surgical hand preparation. AORN J 2000; 71:584-599

39. Doebelling BN, Stanley GL, Sheetz CT, et al: Comparative efficacy of alternative handwashing agents in reducing nosocomial infections in intensive care units. $N$ Engl J Med 1992; 327:88-93

40. Newman JL, Seitz JC: Intermittent use of an antimicrobial hand gel for reducing soapinduced irritation of health care personnel. Am J Infect Control 1990; 18:194-200

41. Bischoff WE, Reynolds TM, Sessler CN, et al: Handwashing compliance by health care workers: The impact of introducing an accessible, alcohol-based hand antiseptic. Arch Intern Med 2000; 160:1017-1021

42. Hugonnet S, Perneger TV, Pittet D: Can alcohol-based handrub improve compliance with hand hygiene in ICU? Abstr. Infect Control Hosp Epidemiol 2000; 21:99

43. Pittet D: Improving compliance with hand hygiene in hospitals. Infect Control Hosp Epidemiol 2000; 21:381-386

44. Voss A, Widmer AF: No time for handwashing? Handwashing versus alcoholic rub: Can we afford 100\% compliance? Infect Control Hosp Epidemiol 1997; 28:205-208

45. Tabor E, Bostwick DC, Evans CC: Corneal damage due to eye contact with chlorhexidine gluconate [letter]. JAMA 1989; 261: 557-558

46. Hamed LM, Ellis FD, Boudreault G, et al: Hibiclens keratitis. Am J Ophthalmol 1987; 104:50-56
47. Garland JS, Alex CP, Mueller CD, et al: Local reactions to a chlorhexidine gluconateimpregnated antimicrobial dressing in very low birth weight infants. Pediatr Infect Dis $J$ 1996; 15:912-914

48. Stables GI, Turner WH, Prescott S, et al: Generalized urticaria after skin cleansing and urethral instillation with chlorhexidinecontaining products. Br J Urol 1998; 82: 756-757

49. Wicki J, Deluze C, Cirafici L, et al: Anaphylactic shock induced by intraurethral use of chlorhexidine. Allergy 1999; 4:768-769

50. Autegarden JE, Pecquet C, Huet S, et al: Anaphylactic shock after application of chlorhexidine to unbroken skin. Contact Dermatitis 1999; 40:215

51. Ebo DG, Stevens WJ, Bridts CH, et al: Contact allergic dermatitis and life-threatening anaphylaxis to chlorhexidine: $J$ Allerg Clin Immunol 1998; 101:128-129

52. Torricelli R. Wuthrich B: Life-threatening anaphylactic shock due to skin application of chlorhexidine [letter]. Clin Exp Allergy 1996; 26:112

53. Okano M, Nomura M, Hata S, et al: Anaphylactic symptoms due to chlorhexidine gluconate. Arch Dermatol 1989; 125:50-52

54. Ophaswongse S, Maibach HI: Alcohol dermatitis: Allergic contact dermatitis and contact urticaria syndrome. Contact Dermatitis 1994; 30:1-6

55. Rilliet A, Hunziker N, Brun R: Alcohol contact urticaria syndrome (immediate-type hypersensitivity). Dermatologica 1980; 161: 361-364

56. Aust LB, Maibach HI: Incidence of human skin sensitization to isostearyl alcohol in two separate groups of panelists. Contact Dermatitis 1980; 6:269-271

57. Waclawski ER, McAlpine LG, Thomson NC: Occupational asthma in nurses caused by chlorhexidine and alcohol aerosols. BMJ 1989; 298:929-930

58. Anonymous: Fire hazard created by the misuse of DuraPrep solution. Health Devices 1998; 27:400-402 